\title{
Cyclin B1: conductor of mitotic symphony orchestra
}

\author{
Huijuan $\mathrm{Yu}^{1}$, Xuebiao Yao ${ }^{1}$ \\ ${ }^{1}$ Laboratory of Cellular Dynamics, Hefei National Laboratory for Physical Science at Microscale; and the University of Science and \\ Technology of China, Hefei, Anhui 230027,China.yaoxb@ustc.edu.cn \\ Cell Research (2008) 18:218-220. doi: 10.1038/cr.2008.20; published online 4 February 2008
}

The inheritance of human genome through mitosis is precisely regulated by a series of tightly orchestrated events such as chromosome condensation, bi-orientated spindle formation, chromosome congression, segregation and cytokinesis. Chromosome movements during mitosis are governed by the interaction of spindle microtubules with a specialized chromosome domain located within the centromere. This specialized region, called the kinetochore, is the site for spindle microtubule-centromere association. In addition to serving as a physical link between chromosomes and spindle microtubules, the kinetochore exhibits an active role in chromosomal segregation through microtubule motors and spindle assembly checkpoint (SAC) sensors located at or near it [e.g., 1].

Several lines of evidence have implicated the kinetochore in generation of a diffusible SAC signal that can block cell cycle progression into anaphase until all kinetochores have successfully attached to spindle microtubules. Aberrant attachment of one or more chromosomes to the spindle is correlated with a corresponding delay in the onset of anaphase. Genetic screen in budding yeast for mutants that fail to arrest the cell cycle in mitosis after disassembly of microtubules, has identified three MAD (mitotic arrest deficiency) and three BUB (budding uninhibited by benomyl) genes $[2,3]$. Vertebrate homologues of MAD1, MAD2, BUB3, BUB1, and BUBR1 are spindle checkpoint components transiently associated with the kinetochore [e.g., 4]

Mitosis is orchestrated by signaling cascades that coordinate mitotic processes and ensure accurate chromosome segregation. The key switch for the onset of mitosis is the archetypal cyclin-dependent kinase Cdk1. Besides the master mitotic kinase Cdk1, three other protein serine/threonine kinase families are also involved, including the Polo kinases, Aurora kinases, and the NEK (NIMA-related kinases) [e.g., 5]. Recent studies have demonstrated that NEK2A interacts with SAC proteins at the kinetochore and plays a vital role in SAC signaling and centromeric cohesion regulation [6].

In mitosis, A- and B-type cyclins bind and activate CDK1 to phosphorylate proteins that mediate the dramatic changes in nuclear and cytoskeletal architecture required for chromosome segregation [e.g., 5]. Even though all CDKs phosphorylate a consensus motif consisting of S/TPxK/R, different cyclin-CDK complexes phosphorylate distinct substrates, raising the question of how substrate specificity is achieved by different cyclin-CDK complexes. At mitotic entry, cyclin B1-CDK1 promotes chromosome condensation, nuclear lamina resolution and mitotic spindle assembly while cyclin B2-CDK1 can only induce Golgi disassembly. It has been proposed that the spatiotemporal dynamics of cyclin B1 and cyclin B2 determines the plasticity of cyclin-CDK on substrate selection. Indeed, cyclin B1 accumulates in the nucleus at prophase
[7], and then at condensed chromatin, spindle microtubules, and centrosomes during prometaphase, corresponding to localizations of known CDK1 substrates, including nuclear lamins and the chromatin-associated protein RCC1. In contrast, cyclin B2 remains associated with the disassembled Golgi during mitosis. Domain mapping studies have suggested that the N-terminal domain is important in dictating the distinct functions of B-type cyclins. Replacement of the cyclin B1 N-terminal domain with that of cyclin B2 is sufficient to mislocalize the chimeric protein to the Golgi [8]. This chimeric protein retains the ability to induce Golgi disassembly, but it loses the ability to induce chromosome condensation, nuclear lamina solubilization, and mitotic aster assembly demonstrating that subcellular localization of different B-type cyclins is a critical determinant of their ability to properly promote the events of mitosis. The SAC determines the anaphase onset by inhibiting the activity of anaphase-promoting complex/cyclosome (APC/C; e.g., reference 4) - an enzyme that adds chains of the small protein ubiquitin to specific substrate proteins, thereby targeting them for destruction. These ubiquitinating cascades are inhibited when unattached kinetochores keep the SAC active. The kinetochores promote the association of $\mathrm{Cdc} 20$ with Mad2 when Cdc20 is phosphorylated by cyclin B-CDK1, which prevents Cdc20 from activating the $\mathrm{APC} / \mathrm{C}$ and hence regulates SAC [9]. Cyclin B-CDK1 
also regulates the kinetochore localization of Plk1 through phosphorylation of the kinetochore proteins Bub1 and INCENP [e.g., reference 4]. Interestingly, CDK1 had been shown to localize to kinetochores during mitosis, but the relevant cyclin partner had remained uncharacterized.

In this issue of Cell Research, Zhang and his colleagues, in an effort to delineate the function of cyclin B1 in mitosis, carried out a thorough analysis of cyclin B1 distribution in mammalian cells [10]. They demonstrated that cyclin B1 is preferentially localized to unattached kinetochores and involved in chromosome alignment in mitosis. In addition, they provided evidence that the CDK1 population at kinetochores is in fact active and that the abundance of the complex varies during mitotic progression. An interesting parallel was recently reported by King's group in which they characterized the precise localization of cyclin B1 using spinning disk confocal microscopy and revealed the structural determinants for spatial distribution of cyclin $\mathrm{B} 1$ to the mitotic apparatus during mitosis [11]. They concluded that separable and distinct mechanisms target cyclin B1 to kinetochores, chromatin, and centrosomes during mitosis. King's group also revealed that CDK1 can target the cyclin B1-CDK1 complex to kinetochores. This mechanism is demonstrated by the localization of cyclin B1 mutants lacking the $\mathrm{N}$-terminal domain that binds to CDK1. Zhang's group further examined the molecular mechanism underlying cyclin B1 localization to the kinetochore and demonstrated the function of the dynein/dynactin complex in regulating the dynamics of cyclin B1 on kinetochores. In addition, Zhang's group revealed that cyclin B1 is translocated from kinetochores toward spindle poles along microtubules when the kinetochores are properly attached and the SAC is inactivated.

Interestingly, cyclin B1 localization to the outer kinetochore during prometaphase depends on both Hec1 and Mad2 as either depletion abolishes kinetochore localization of cyclin B1. Since it has never been shown that Mad2 is essential for localization of other checkpoint proteins, it would be of great significance to delineate the underlying mechanisms accounting for the dependencies of cyclin B1 on Mad2. Given the fact that non-degradable cyclin B1 exhibits normal turnover dynamics at the kinetochore, these new data argue that $\mathrm{Cdc} 20$ or $\mathrm{APC} / \mathrm{C}$-association alone is sufficient to recruit cyclin $\mathrm{B} 1$ to kinetochores.

The findings of cyclin B1 enriched at unattached kinetochores of mammalian cells are consistent with the early hypothesis of a positive role of CDK1 in SAC activity. One potential function of kinetochore-associated cyclin B1CDK1 could be to promote inhibition of Cdc2 20 by Mad2. Cdc20 contains two putative Cdk1 phosphorylation sites, and $\mathrm{Cdc} 20$ phosphorylation is required for Mad2 binding, as nonphosphorylatable versions of $\mathrm{Cdc} 20$ are resistant to Mad2 binding and inhibition [9, 12]. Cdc20 localizes to kinetochores from prophase through anaphase, but its association is highly dynamic. It is likely that localization of cyclin B1-CDK1 to unattached kinetochores could enable the local phosphorylation of $\mathrm{Cdc} 20$, promoting its binding to the active conformation of Mad2 generated at the kinetochore. In addition, other kinetochore proteins are known substrates of the cyclin B1-CDK1 complex, including INCENP, Bub1, and APC/C subunits. Therefore, recruitment of cyclin B1-CDK1 to unattached kinetochores could affect the activity of other SAC components or regulate other signaling events essential for anaphase onset. Given the recent identification of the deubiquitinating enzyme USP44 at the kinetochore by Elledge and Kirschiner teams [13], the current study by Zhang's group raises the possibility that phosphorylation and deubiquitination may modulate the molecular plasticity of
Cdc20 in SAC signaling. In sum, the findings of cyclin B1-CDK1 dynamics in the mitotic apparatus provide a new direction in delineating the SAC signaling cascade.

\section{References}

1 Yao X, Abrieu A, Zheng Y, Sullivan KF, Cleveland DW. CENP-E forms a link between attachment of spindle microtubules to kinetochores and the mitotic checkpoint. Nat Cell Biol 2000; 2:484-491.

2 Li R, Murray AW. Feedback control of mitosis in budding yeast. Cell 1990; 66:519-531.

3 Hoyt MA, Totis L, Roberts BTS. S. cerevisiae genes required for cell cycle arrest in response to loss of microtubule function. Cell 1990; 66:507-517.

4 Musacchio A, Salmon ED. The spindleassembly checkpoint in space and time. Nat Rev Mol Cell Biol 2007; 8:379. 393.

5 Nigg EA. Mitotic kinases as regulators of cell division and its checkpoints. Nat Rev Mol Cell Biol 2001; 2:21-32.

6 Fang L, Fang G. Centromere cohesion: regulating the guardian. Cell Res 2007; 17:664-665.

7 Pines J, Hunter T. The differential localization of human cyclins A and B is due to a cytoplasmic retention signal in cyclin B. EMBO J 1994; 13:37723781.

8 Draviam VM, Orrechia S, Lowe M, Pardi R, Pines J. The localization of human cyclins B1 and B2 determines CDK1 substrate specificity and neither enzyme requires MEK to disassemble the Golgi apparatus. J Cell Biol 2001; 152:945-958.

9 D'Angiolella V, Mari C, Nocera D, Rametti L, Grieco D. The spindle checkpoint requires cyclin-dependent kinase activity. Genes Dev 2003; 17:25202525

10 Chen Q, Zhang X, Jiang Q et al. Cyclin B1 is localized to unattached kinetochores and contributes to efficient microtubule attachment and proper chromosome alignment during mitosis. Cell Res 2008; 18:268-280.

11 Bentley AM, Normand G, Hoyt J, King RW. Distinct sequence elements of Cyclin B1 promote localization to chromatin, centrosomes, and kinetochores 
during mitosis. Mol Biol Cell 2007; 18:4847-4858

12 Chung E, Chen RH. Phosphorylation of Cdc20 is required for its inhibition by the spindle checkpoint. Nat Cell Biol 2003; 5:748-753.

13 Stegmeier F, Rape M, Draviam VM, et al. Anaphase initiation is regulated by antagonistic ubiquitination and deubiquitination activities. Nature 2007;

446:876-880. 